\title{
Sequent depth ratio of B-jumps on smooth and rough beds
}

\author{
Francesco Giuseppe Carollo, Vito Ferro, Vincenzo Pampalone \\ Dipartimento di Scienze Agrarie e Forestali, Università di Palermo, Italy
}

\begin{abstract}
A hydraulic B-jump has the toe section located on a positively sloping upstream channel and the roller end on a downstream horizontal channel. This paper analyses the B-jump on a rough bed, such as at the transition from a block ramp to the stilling basin. Laboratory measurements of the sequent depth were carried out using three different channel slopes for the rough bed and a single slope for the smooth bed. A solution useful for estimating the sequent depth ratio in a rectangular channel for different relative roughness and bed slope is proposed and positively tested by the present measurements. This solution can also be used to estimate the sequent depth ratio of classical hydraulic jumps or B-jumps on smooth and rough beds.
\end{abstract}

\section{Introduction}

For its high turbulence, the hydraulic jump is widely used as an energy dissipator in hydraulic structures. The sloping jump, i.e. the hydraulic jump in sloping channels, is often seen in hydraulic structures as, for example, on block ramps. The knowledge of both the sequent depth ratio and the length of the jump is required in order to

Correspondence: Francesco Giuseppe Carollo, Dipartimento di Scienze Agrarie e Forestali, Università di Palermo, viale delle Scienze, 90128 Palermo, Italy.

Tel. +39.091 .23897064 - Fax: +39.091 .484035 .

E-mail: francesco_giuseppe.carollo@unipa.it

Key words: open channel flow, hydraulic jump, energy dissipation, sloping channel, bed roughness.

Contributions: the authors contributed equally.

Conflict of interests: the authors declare no potential conflict of interests.

Funding: the research was funded by financial support of Governo Italiano, MIUR 60\%, 2007.

Received for publication: 13 June 2013.

Accepted for publication: 10 September 2013.

(C) Copyright FG. Carollo et al., 2013

Licensee PAGEPress, Italy

Journal of Agricultural Engineering 2013; XLIV:e12

doi:10.4081/jae.2013.e12

This article is distributed under the terms of the Creative Commons Attribution Noncommercial License (by-nc 3.0) which permits any noncommercial use, distribution, and reproduction in any medium, provided the original author(s) and source are credited. design the overall hydraulic structure composed of the block ramp and the stilling basin.

A classification of sloping jumps was introduced by Kindsvater (1944). The present investigation refers to the B-jump, having its toe on the sloping bed and the end of roller at the tailwater horizontal bed. For sloping jumps, the one-dimensional (1-D) momentum equation cannot be applied to determine the sequent depth ratio because additional information is needed to compute both the weight component of the jump (Gunal and Narayanan, 1996) and the bottom pressure acting on the sloping channel portion (Carollo et al., 2011).

One of the first investigations concerning the B-jump on a smooth bed was carried out by Bradley and Peterka (1957) for angles $\alpha$ from $2.9^{\circ}$ to $16.7^{\circ}$ of the upstream sloping bed. Hager (1988) investigated Bjumps in a channel with $\alpha=45^{\circ}$ and Kawagoshi and Hager (1990) extended the previous findings to $\alpha=30^{\circ}$. According to these Authors, the sequent depth ratio depends on the channel slope, the approach flow Froude number:

$$
\mathrm{F}_{1}=\frac{Q \cos \alpha}{L h_{1} \sqrt{g h_{1}}}
$$

where:

$Q$ is discharge, $L$ is channel width, $h_{1}$ is pressure head at jump toe section and $g$ is gravity acceleration, with parameter $E$ accounting for the jump toe position as:

$$
E=\frac{h_{2}-z_{1}}{h_{2}}
$$

where:

$h_{2}$ is the subcritical sequent depth and $z_{1}$ the bottom elevation at the toe section above the horizontal tailwater bottom (Figure 1). Other laboratory investigations were conducted by Ohtsu and Yasuda (1990, 1991) and Adam et al. (1993). Beirami and Chamani (2006) investigated the B-F jump, which begins on a positive slope and ends on an adverse sloping channel. However, information concerning the effects of bed roughness on the B-jump is still lacking.

This paper reports the results of a laboratory investigation aimed to establish the sequent depth ratio of B-jumps in a rough channel. Its aim is to extend the analytical findings of two previous papers dealing with hydraulic jumps on horizontal rough and smooth beds (Carollo et al., 2009) and B-jumps on a smooth bed (Carollo et al., 2011), respectively. A solution for estimating the sequent depth ratio of various relative roughness and bed slope conditions is the synthesis of two relationships, previously proposed by the Authors and positively tested by the present data.

\section{Experimental installation}

The experimental tests were conducted at the Dipartimento di 
Scienze Agrarie e Forestali, University of Palermo, Italy. The flume, with glass walls and bed, was $4.90 \mathrm{~m}$ long, $0.304 \mathrm{~m}$ wide and $0.24 \mathrm{~m}$ deep, and was connected to a hydraulic circuit allowing for recirculation of a steady discharge. The discharge $Q$ was measured by an electro-magnetic flow meter to a maximum error of $\pm 0.2 \%$. Angles of $\alpha=8.5^{\circ}, 12^{\circ}$ and $17.5^{\circ}$ were provided on a smooth plane, $0.50 \mathrm{~m}$ or $0.70 \mathrm{~m}$ long, over which closely packed crushed gravel particles were fixed. The rough horizontal bed contained gravel particles cemented to a plate placed on the flume bottom and extended $1.20 \mathrm{~m}$ downstream of the sloping plane. A single bed roughness was tested. The grain-size distribution was obtained by a sample of 100 particles. For each particle, three axial sizes were measured and its diameter $d$ was calculated as the mean value. The grain-size distribution was almost uniform with $d_{50}=0.54 \mathrm{~cm}$ and the geometric standard deviation (Pagliara et al., 2008) of $s=\left(d_{84} / d_{16}\right)^{0.5}=1.14$, with $d_{\mathrm{xx}}$ as diameter of the bed particles for which $\mathrm{xx} \%$ are finer. The median diameter $d_{50}$ was assumed as roughness height $k_{s}$. Additional experimental runs for a smooth bed with $\alpha=12^{\circ}$ were also conducted.

For a given discharge, the tailwater level was adjusted by a hydraulic bar screen located at the channel outlet so that the jump was entirely set in the experimental channel portion at different elevations on the sloping plane. The use of the hydraulic bar screen determined a nearly horizontal surface profile upstream of the channel end section. The vertical flow depths $\mathrm{h}_{1}{ }_{1}$ (Figure 1) at the toe section and $h_{2}$ were measured by a point gauge of $\pm 0.1 \mathrm{~mm}$ reading accuracy. The pressure head $h_{1}$ was calculated as $h_{1}=h^{*}{ }_{1} \cos ^{2} \alpha$. Because of the possible streamline curvature due to the slope change at the end of the chute, for low $z_{1}$ the head pressure may be greater than the hydrostatic value $\mathrm{h}^{*}{ }_{1} \cos ^{2} \alpha$, especially for the highest slopes. However, for all runs, no streamline curvature was visually appreciable at the end of the chute and the hydrostatic hypothesis on $h_{1}$ was always assumed. For B-jumps on a smooth bed, the flow depths were measured at the channel axis. For Bjumps on a rough bed, the jump had nearly a non-developed roller flow (Hager, 1992) in which a small counter-clockwise vortex followed a large clockwise vortex with a horizontal axis. To accurately locate the toe section of the jump, the supercritical flow profile along the sloping plane was first drawn on the channel sidewall. Then, after the jump was set over the rough bed, the toe section was chosen at the last section, in the flow direction, where the surface profile still agreed with the profile drawn on the channel sidewall.

Since the supercritical approach flow had a flow depth that was not constant within the cross-section (due to the effect of bed roughness elements), $h_{l}^{*}$ was measured at the flume axis and near the two side walls. The average of the three values was considered for data analysis. The sequent flow depth $h_{2}$ was measured axially downstream of the end of the roller where the surface profile was nearly horizontal. The end of the roller was determined by visualising the characteristic stagnation point with a float. All actual flow depths were calculated as the distance between the water surface and the virtual bed level (reference plane) (Figure 1) that was located $0.35 k_{s}$ below the horizontal plane passing at the particle tops (Bayazit, 1976). The sequent depth ratio was calculated as $Y=h_{2} / h_{1}$. For each run, the horizontal distance $l$ (Figure 1) between the toe section of the jump and the toe of the sloping plane was also measured.

The ranges of the variables $\alpha, E, h_{1}, \mathrm{~F}_{1}, Y$ and $k_{s} k$, where $k=\left(Q^{2} / g L^{2}\right)^{1 / 3}=$ critical flow depth, characterising the experimental data are listed in Table 1.

For fluid flows, scale effects occur if the flow regime is different between model and prototype. As the investigated flows are typically turbulent, the absence of scale effects is verified if the used model assures turbulent flows. According to De Marchi (1986), the flow is fully turbulent if the shear Reynolds number $\operatorname{Re}_{1}{ }^{*}=\left(g h^{*}{ }_{1} \sin \alpha\right)^{0.5} k_{s}{ }^{-1}>70$, where $v=$ kinematic viscosity. Chanson (1999) and Henderson (1966) fixed this value of $R e_{1}{ }^{*}$ at 100 . The present data on the rough bed were characterised by $\operatorname{Re}_{1}{ }^{*}>630$, so that no scale effects should have occurred. According to Leutheusser and Kartha (1972), taking into account that the measurements were carried out for $F_{1}<10$, the effect of inflow condition on the sequent depth ratio was neglected.

\section{Results and discussion}

According to Carollo et al. (2011), a B-jump on the rough bed is expressed by:

$$
F\left(g, \rho, \mu, h_{1}, h_{2}, z_{1}, Q, L, k_{s}, \alpha\right)=0
$$

where:

$F$ is a functional symbol, $\rho$ is water density, and $\mu$ is water viscosity. According to the $\Pi$ theorem of dimensional analysis (Barenblatt, 1987), choosing $h_{1}, g$ and $\rho$ as dimensional independent variables and rearranging the dimensionless deduced groups as suggested by Carollo et al. (2009), Eq. 3 becomes:

$$
Y-\frac{1}{E}=\phi\left(\mathrm{F}_{1}-1, \frac{h_{1}}{L}, \operatorname{Re}_{1}^{*}, \frac{k_{s}}{k}, E, \alpha\right)
$$

where:

$R e_{1}^{*}=Q \rho L^{-1} \mu^{-1}$ is the approach flow modified Reynolds number.

Neglecting the dependence of $Y$ on $h_{1} / L$ and $R e_{1}{ }^{*}$ as suggested by Carollo et al. (2009) Eq. 4 can be rewritten as:

$$
Y-\frac{1}{E}=\phi\left(\mathrm{F}_{1}-1, \frac{k_{s}}{k}, E, \alpha\right)
$$

where $\phi$ is a functional symbol.

Carollo et al. $(2009,2011)$ experimentally verified the incomplete self-similarity hypothesis regarding the group $\mathrm{F}_{1}-1$ obtaining:

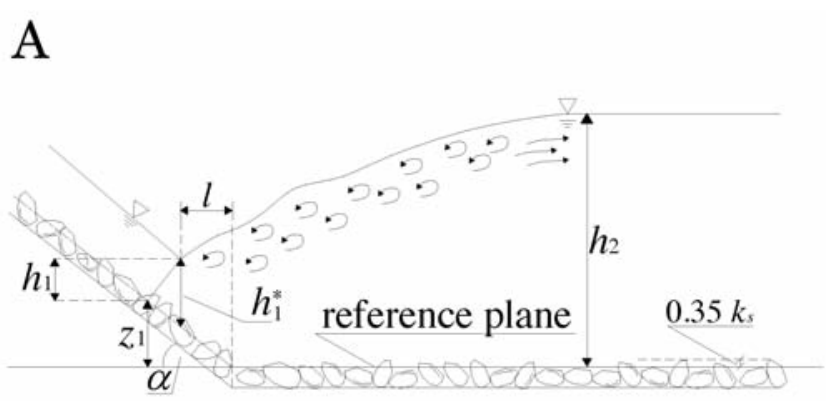

B

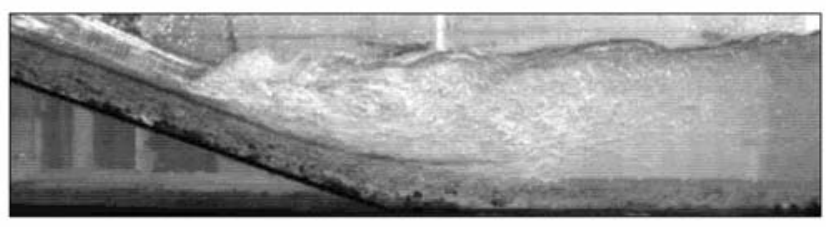

Figure 1. Sketch (A) and view (B) of a B-jump on a rough bed. 


$$
Y-\frac{1}{E}=m\left(\frac{k_{s}}{k}, E, \alpha\right)\left(\mathrm{F}_{1}-1\right)^{0.963}
$$

where:

$m$ is a functional symbol depending on relative roughness, the jump position and the angle $\alpha$. Eq. 6 satisfies the boundary condition $Y=1 / E$ if $F_{1}=1$. For $F_{1}=1$ the chute slope is critical and the free surface steady profile downstream of $h_{1}$ is horizontal. Assuming that the water depth $h_{2}$ is located where the above horizontal surface profile ends, the following equation is valid:

$$
h_{2}=h_{1}+z_{1}
$$

Introducing Eq. 7 into Eq. 2 results in $Y=1 / E$.

For a hydraulic jump on a horizontal $\left(E=1, \alpha=0^{\circ}\right)$ smooth or rough bed, Carollo et al. (2009) proposed, in agreement with Eq. 6:

$$
Y-1=\sqrt{2} \exp \left(-\frac{k_{s}}{k}\right)\left(\mathrm{F}_{1}-1\right)^{0.963}
$$

establishing an incomplete self-similarity condition regarding the dimensionless group $\exp \left(k_{d} / k\right)$. For a horizontal smooth bed condition $\left(k_{s} / k=0\right)$, Carollo et al. (2009) verified that Eq. 8 practically coincides with Bresse's solution for $F_{1}<8$ while, for $F_{1}>8$, Eq. 8 gives sequent depth ratios less than those obtained by Bresse's solution and in agreement with the experimental data. This discrepancy could be attributed to the neglect of the boundary channel resistance in the Bresse solution or to the effect of Froude number on air entrainment (Carollo et al., 2009). For a B-jump on a hydraulically smooth bed, Carollo et al. (2011) verified that, according to Eq. 6, the following empirical relationship:

$$
\mathrm{Y}=\sqrt{2} \exp \left(-\frac{\tan \alpha}{\mathrm{a}}\right) \mathrm{E}^{-\frac{\mathrm{b}}{(\tan \alpha)^{c}}}\left(\mathrm{~F}_{1}-1\right)^{0.963}+\frac{1}{\mathrm{E}}
$$

where:

$a, b$ and $c$ are three coefficients, is applicable. Using the 103 tests of Bradley and Peterka (1957), characterised by $\alpha=2.9^{\circ}, 5.7^{\circ}, 8.5^{\circ}, 11.3^{\circ}$, $14^{\circ}, 16.7^{\circ}$, and the 219 present tests for $\alpha=8.5^{\circ}, 17.5^{\circ}$ and $30^{\circ}$, Carollo et al. (2011) obtained $a=8.42, b=0.181$ and $c=0.663$.

For a smooth bed, Eq. 9 with the above values of $a, b$ and $c$ yields systematic underestimates of $Y$ values in comparison with both the 72 tests carried out here for $\alpha=12^{\circ}$, and the 45 tests of Bradley and Peterka (1957) for $\alpha=11.3^{\circ}$ and $14^{\circ}$. To improve its reliability, Eq. 9 was recalibrated to:

$$
\mathrm{Y}=\sqrt{2} \exp \left(-\frac{\tan \alpha}{11.01}\right) \mathrm{E}^{-\frac{0.202}{(\tan \alpha)^{0.64}}}\left(\mathrm{~F}_{1}-1\right)^{0.963}+\frac{1}{\mathrm{E}}
$$

The experimental $m$ values

$$
m=\frac{Y-\frac{1}{E}}{\left(\mathrm{~F}_{1}-1\right)^{0.963}}
$$

are plotted versus $E$, as an example for $\alpha=17.5^{\circ}$ (Figure 2). The measurements, arranged for $k_{d} / k$ intervals, indicate that $m$ decreases as $E$ increases and, for a fixed $E$ value, $m$ slightly decreases as the relative roughness increases. A comparison between the rough and hydraulically smooth bed conditions shows that boundary roughness reduces $m$ and, according to Eq. 6, the sequent depth ratio. This result supports the findings of previous investigations (Carollo et al., 2007, 2009; Hughes and Flack, 1984; Ead and Rajaratnam, 2002; Pagliara et al., 2008). The present data for rough beds, plotted in Figure 3, allow us to assess the effects of $\alpha$ on the coefficient $m$. Accordingly, $m$ increases as $\alpha$ decreases from $17.5^{\circ}$ to $12^{\circ}$, while there is no appreciable effect for $\alpha=12^{\circ}$ and $\alpha=8.5^{\circ}$. Using the present tests for the rough bed, the sequent depth ratio follows as:

$$
Y=\sqrt{2} \exp \left(-\frac{k_{s}}{k}\right) \exp \left(-\frac{\tan \alpha}{11.01}\right) E^{-\frac{0.202}{(\tan \alpha)^{0.54}}}\left(\mathrm{~F}_{1}-1\right)^{0.963}+\frac{1}{E}
$$

that agrees with both Eq. 8 (for $E=1$ and $\alpha=0^{\circ}$ ) and Eq. 10 (for $k_{s} / k=0$ ). Thus, Eq. 12 can be used to estimate the sequent depth ratio of classic hydraulic jumps or B-jumps.

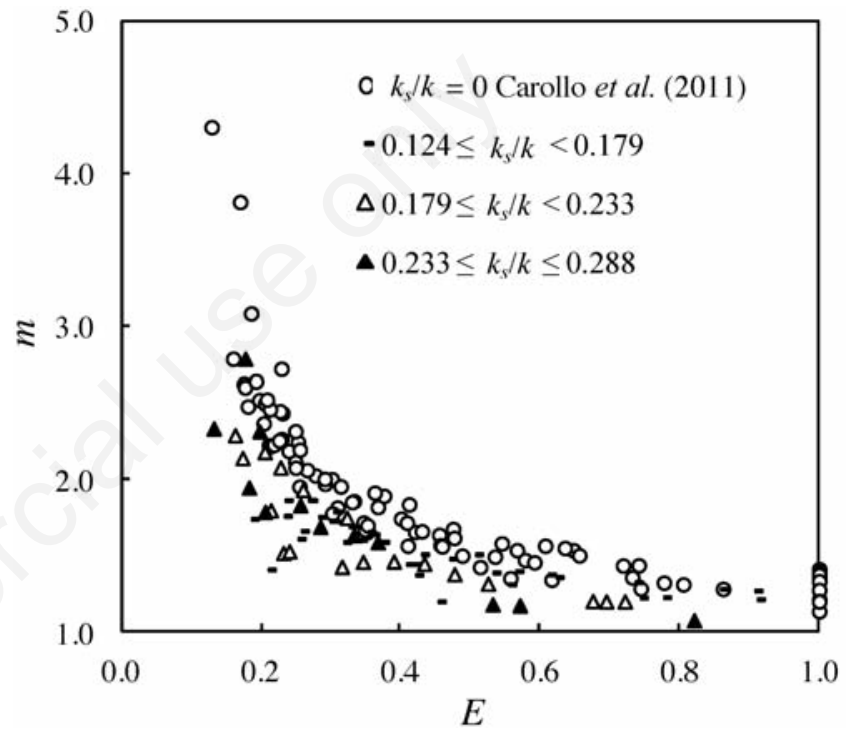

Figure 2. Plot of the experimental pairs $(E, m)$ arranged at intervals of $k_{s} / k$ for $\alpha=17.5^{\circ}$.

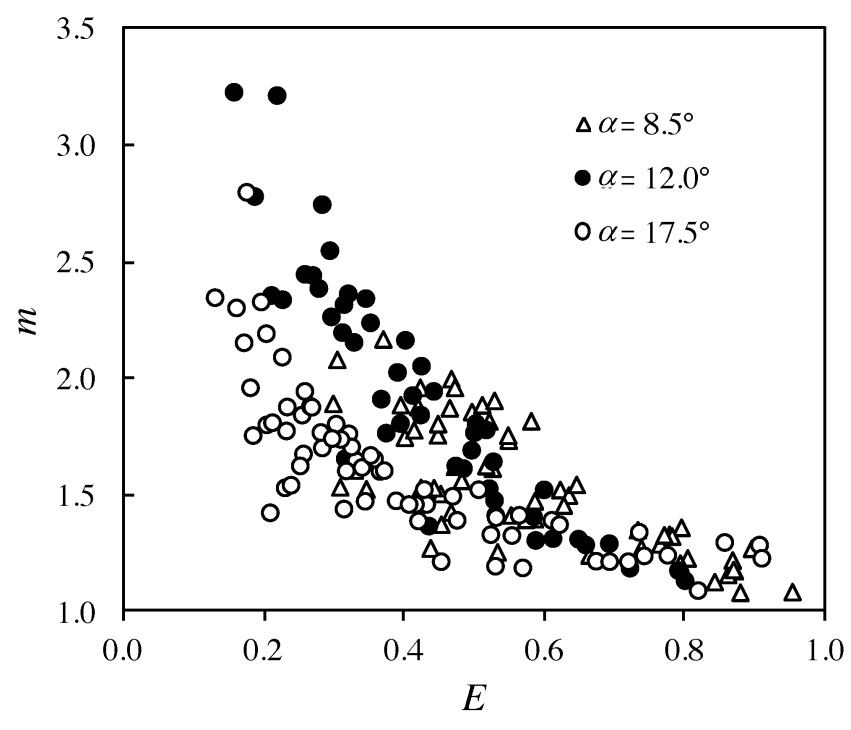

Figure 3. Plot of all experimental pairs $(E, m)$ corresponding to $\alpha=8.5^{\circ}, 12^{\circ}$ and $17.5^{\circ}$. 
Table 1. Range of application of experimental runs.

\begin{tabular}{|c|c|c|c|c|c|c|}
\hline $\begin{array}{l}\alpha \\
\left({ }^{\circ}\right)\end{array}$ & $\begin{array}{c}\text { No. of experimental } \\
\text { Runs }\end{array}$ & $\begin{array}{l}E \\
-\end{array}$ & $\begin{array}{l}h_{1} \\
\mathrm{~cm}\end{array}$ & $\begin{array}{c}F_{1} \\
-\end{array}$ & $\begin{array}{l}Y \\
-\end{array}$ & $k s / k$ \\
\hline 12.0 & 72 & $0.18-1.00$ & $0.52-2.18$ & $2.11-6.11$ & $5.82-18.13$ & 0.00 \\
\hline 8.5 & 60 & $0.30-0.96$ & $1.02-3.06$ & $1.76-2.99$ & $2.97-6.46$ & $0.12-0.25$ \\
\hline 12.0 & 48 & $0.16-0.80$ & $0.66-2.28$ & $2.64-4.51$ & $4.76-15.35$ & $0.12-0.29$ \\
\hline 17.5 & 66 & $0.13-0.91$ & $0.63-2.18$ & $2.71-4.86$ & $4.80-14.56$ & $0.12-0.29$ \\
\hline
\end{tabular}

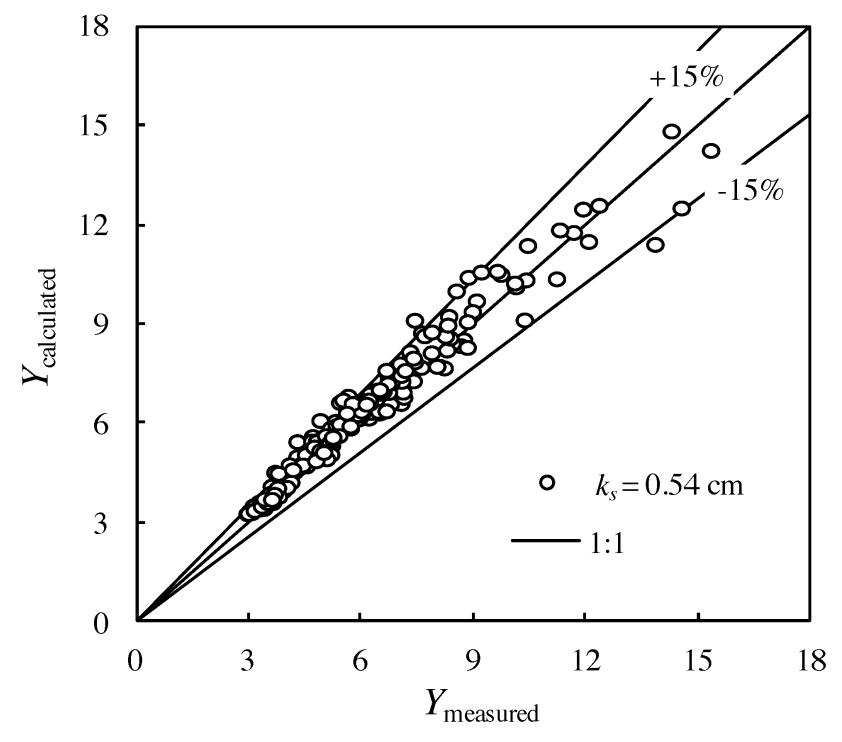

Figure 4. Comparison between the measured values of the sequent depth ratio $Y$ and those calculated by Eq. 12 .

A comparison between the measured values of the sequent depth ratio $Y$ and calculated by Eq. 12 is plotted in Figure 4. Eq. 12 has a mean error of $\pm 7.8 \%$ with only 16 data of the $174 Y$ ratios falling out of the error band of $\pm 15 \%$. The detected uncertainty of $\pm 15 \%$ on $Y$ is probably due to the difficulty in determining the supercritical flow depth because of its variability along the cross-section due to the effect of bed roughness and the uncertainty on the reference level location (where $\left.h_{1}=0\right)$.

Use of only Eq. 12 is not sufficient to establish the sequent depth ratio because the dimensionless parameter $E$ is unknown. To solve this problem, Eq. 12 has to be used jointly with the steady supercritical surface profile $h_{1}\left(z_{1}\right)$ on the sloping bed (Carollo et al., 2011).

Because of the empirical origin of Eq. 12, the present results are applicable within the tested experimental range (Table 1).

\section{Conclusions}

A B-jump usually occurs at the lower portion of hydraulic structures such as spillways or block ramps where the channel bottom varies from sloping to horizontal. This paper reports the results of a laboratory investigation carried out using a smooth sloping bed and three different sloping beds of identical roughness. A dimensionless functional relationship of the sequent depth ratio for B-jumps on a rough bed was introduced. In two previous papers, dealing with hydraulic jumps on smooth or rough horizontal beds and B-jumps on the smooth bed, the use of the incomplete self-similarity theory allowed us to determine that the sequent depth ratio is a power function of the group $\left(F_{1}-1\right)$, whereas the scale coefficient $m$ depends on the others dimensionless groups.

Experiments indicate that $m$ decreases as $E$ increases and, for a given $E$ value, $m$ slightly decreases as the relative roughness increases. In comparison with a hydraulically smooth bed condition, the bed roughness reduces the sequent depth ratio. The analysis showed that $m$ increases as $\alpha$ decreases from $17.5^{\circ}$ to $12^{\circ}$, while no appreciable effect was observed for $\alpha=12^{\circ}$ and $8.5^{\circ}$. An empirical relationship for estimating $m$ by bottom slope, toe section position and relative roughness was also presented. Therefore, the solution, Eq. 12, to estimate the sequent depth ratio was obtained as a synthesis of Eqs. 8 and 10 and positively tested by the available data on rough beds. This solution can be used to estimate the sequent depth ratio of hydraulic jumps on various relative roughness and bed slope conditions. Since Eq. 12 is empirically derived, it is valid within the tested experimental range and further investigations with different experimental configurations should be carried out to confirm its reliability.

\section{Main symbol list}

$a=$ coefficient of Eq. 9;

$b=$ coefficient of Eq. 9;

$c=$ coefficient of Eq. 9;

$d=$ diameter of the bed particles;

$d_{\mathrm{xx}}=$ diameter of the bed particles for which $\mathrm{xx} \%$ are finer;

$E=\left(h_{2}-z_{1}\right) / h_{2}$;

$F=$ functional symbol of Eq. 3;

$\mathrm{F}_{1}=$ approach flow Froude number;

$g$ = gravity acceleration;

$h_{1}^{*}=$ flow depth vertically measured at jump toe section;

$h_{1}=$ pressure head at jump toe section;

$h_{2}=$ downstream subcritical flow depth;

$k=$ critical flow depth;

$k_{s}=$ roughness height;

$l=$ horizontal distance between jump toe and chute end section;

$L=$ channel width;

$m=$ coefficient;

$Q=$ discharge;

$R e_{1}{ }^{*}=$ inflow shear Reynolds number;

$Y=$ sequent depth ratio $h_{2} / h_{1}$;

$z_{1}=$ bottom elevation at jump toe section;

$\alpha=$ angle of upstream sloping bed relative to horizontal;

$\phi=$ functional symbol of Eq. 4 ;

$v$ = kinematic viscosity;

$\sigma=\left(\delta_{84} / d_{16}\right)^{0.5}$ coefficient of geometrical standard deviation. 


\section{References}

Adam A.M., Ruff J.F., AlQaser G., Abt S.R. 1993. Characteristics of Bjump with different toe locations. J. Hydraul. Eng. ASCE. 119:93848.

Bayazit M. 1976. Free surface flow in a channel of large relative roughness. J. Hydraul. Res. 14:115-25.

Barenblatt G.I. 1987. Dimensional analysis. Gordon and Breach Science, Amsterdam, The Netherlands.

Beirami M.K., Chamani M.R. 2006. Hydraulic jumps in sloping channels: sequent depth ratio. J. Hydraul. Eng. ASCE. 132:1061-8.

Bradley J.N., Peterka A.J. 1957. Hydraulic design of stilling basins: stilling basin with sloping apron (basin V). J. Hydr. Eng. Div. ASCE. 83:1-32.

Carollo F.G., Ferro V., Pampalone V. 2007. Hydraulic jumps on rough beds. J. Hydraul. Eng. ASCE. 133:989-99.

Carollo FG, Ferro V, Pampalone V. 2009. New solution of classical hydraulic jump. J. Hydraul. Eng. ASCE. 135:527-31.

Carollo F.G., Ferro V., Pampalone V. 2011. Sequent depth ratio of a B jump. J. Hydraul. Eng.-ASCE. 137: 651-58.

Chanson H. 1999. The hydraulics of open channel flow. Arnold, London, UK.

De Marchi G. 1986. Idraulica, vol. 1, parte 1ª 3 th ed. ED. Hoepli, Milano,
Italy.

Ead S.A., Rajaratnam N. 2002. Hydraulic jump on corrugated beds. J. Hydraul. Eng. ASCE. 128:656-63.

Gunal M., Narayanan R. 1996. Hydraulic jump in sloping channels. J. Hydraul. Eng. ASCE. 122:436-42.

Hager W.H. 1988. B-jump in sloping channel. J. Hydraul. Res. 26:539 58.

Hager W.H. 1992. Energy dissipators and hydraulic jump. Kluver Academic, Dordrecht, the Netherlands.

Henderson F.M. 1966. Open channel flow. Macmillan, New York, USA.

Hughes W.C., Flack J.E. 1984. Hydraulic jump properties over a rough bed. J. Hydraul. Eng. ASCE. 110:1755-71.

Kawagoshi N., Hager W.H. 1990. B-jump in sloping channel, II. J. Hydraul. Res. 28:461-80.

Kindsvater C.E. 1944. The hydraulic jump in sloping channels. Trans. ASCE. 109:1107-20.

Leutheusser H.J., Kartha V.C. 1972. Effect of inflow condition on hydraulic jump. J. Hydr. Eng. Div. ASCE. 98:1367-85.

Ohtsu I., Yasuda Y. 1990. Discussion of B-jump in sloping channel, by Hager WH. J. Hydraul. Res. 28:105-19.

Ohtsu I., Yasuda Y. 1991. Hydraulic jump in sloping channels. J. Hydraul. Eng. ASCE. 117:905-21.

Pagliara S., Lotti I., Palermo M. 2008. Hydraulic jump on rough bed of stream rehabilitation structures. J. Hydroenviron. Res. 2:29-38. 